Interface Components: Nanoparticles, Colloids, Emulsions, Surfactants, Proteins, Polymers

\title{
On the time and mechanism of nanoparticle functionalization by macromolecular ligands during pulsed laser ablation in liquids
}

Alexander Letzel, Stefan Reich, Tomy dos Santos Rolo, Alexander Kanitz, Jan Hoppius, Alexander Rack, Margie Olbinado, Andreas Ostendorf, Bilal Gökce, Anton Plech, and Stephan Barcikowski

Langmuir, Just Accepted Manuscript • DOI: 10.1021/acs.langmuir.8b01585 • Publication Date (Web): 15 Jan 2019

Downloaded from http://pubs.acs.org on January 17, 2019

\section{Just Accepted}

"Just Accepted" manuscripts have been peer-reviewed and accepted for publication. They are posted online prior to technical editing, formatting for publication and author proofing. The American Chemical Society provides "Just Accepted" as a service to the research community to expedite the dissemination of scientific material as soon as possible after acceptance. "Just Accepted" manuscripts appear in full in PDF format accompanied by an HTML abstract. "Just Accepted" manuscripts have been fully peer reviewed, but should not be considered the official version of record. They are citable by the Digital Object Identifier (DOI®). "Just Accepted" is an optional service offered to authors. Therefore, the "Just Accepted" Web site may not include all articles that will be published in the journal. After a manuscript is technically edited and formatted, it will be removed from the "Just Accepted" Web site and published as an ASAP article. Note that technical editing may introduce minor changes to the manuscript text and/or graphics which could affect content, and all legal disclaimers and ethical guidelines that apply to the journal pertain. ACS cannot be held responsible for errors or consequences arising from the use of information contained in these "Just Accepted" manuscripts. 


\section{On the time and mechanism of nanoparticle}

\section{functionalization by macromolecular ligands during}

\section{pulsed laser ablation in liquids}

Alexander Letzel, ${ }^{1 \ddagger}$ Stefan Reich, ${ }^{2 \ddagger}$ Tomy dos Santos Rolo, ${ }^{2}$ Alexander Kanitz, ${ }^{3}$ Jan Hoppius, ${ }^{3}$ Alexander Rack, ${ }^{4}$ Margie Olbinado, ${ }^{4}$ Andreas Ostendorf, ${ }^{3}$ Bilal Gökce, ${ }^{1 *}$ Anton Plech, ${ }^{2 *}$ and Stephan Barcikowski ${ }^{1}$

${ }^{1}$ Department of Technical Chemistry I and Center for Nanointegration Duisburg-Essen (CENIDE), University of Duisburg-Essen, Universitätsstr. 7, 45141 Essen, Germany

${ }^{2}$ Institute for Photon Science and Synchrotron Radiation, Karlsruhe Institute of Technology (KIT), Hermann-von-Helmholtz-Platz 1, 76344 Eggenstein-Leopoldshafen, Germany

${ }^{3}$ Applied Laser Technologies, Ruhr-University Bochum, Universitätsstr. 150, 44801 Bochum, Germany

${ }^{4}$ European Synchrotron Radiation Facility (ESRF), 30843 Grenoble, France

\section{KEYWORDS}

X-ray imaging; Hartmann mask; Laser ablation synthesis in solution; Size quenching; In situ 


\begin{abstract}
Laser ablation of gold in liquids with nanosecond laser pulses in aqueous solutions of inorganic electrolytes and macromolecular ligands for gold nanoparticle size quenching is probed inside the laser-induced cavitation bubble by in situ X-ray multi-contrast imaging with a Hartmann mask (XHI). It is found that (i) the in situ size quenching power of sodium chloride ( $\mathrm{NaCl})$ in comparison to the ablation in pure water can be observed by the scattering contrast from XHI already inside the cavitation bubble, while (ii) for polyvinylpyrrolidone (PVP) as a macromolecular model ligand an in situ size quenching cannot be observed. Complementary ex situ characterization confirms the overall size quenching ability of both additive types $\mathrm{NaCl}$ and PVP. The macromolecular ligand as well as its monomer N-vinylpyrrolidone (NVP) are mainly effective for growth quenching of larger nanoparticles on later time scales, leading to the conclusion of an alternative interaction mechanism with ablated nanoparticles compared to the electrolyte $\mathrm{NaCl}$, probably outside of the cavitation bubble, in the surrounding liquid phase. While monomer and polymer have similar effects on the particle properties, with the polymer being slightly more efficient, only the polymer is effective against hydrodynamic aggregation.
\end{abstract}

\title{
INTRODUCTION
}

Since the discovery of pulsed laser ablation in liquids (LAL) as a tool for the synthesis of nanoparticles in 1993 [1] researchers still face major challenges that hinder LAL from being established as a competitive synthesis route towards nanomaterials [2]. Two of these are the control of mean size, and size distribution width. The challenge of gaining control over the final 
nanoparticle size in a single-step approach and without using surfactants or ligands was accelerated by the discovery that micromolar concentrations of monovalent salts like sodium chloride $(\mathrm{NaCl})$ result in a size quenching of gold nanoparticles by adsorption to the particle surface and stronger electrostatic stabilization $[3,4]$. This approach was extended to other noble metals like palladium [5] and platinum [6]. It was found recently that size reduction by ions already happens inside the gas phase [7] of the laser-induced cavitation bubble [8-10] some tens of microseconds after a laser pulse hits the target [7]. Ions present in the electrolyte get in contact very early to the plasma emitted from the target and are thus able to interact with the material even before bubble expansion [11]. Note that Sakka et al. [12] demonstrated the presence of dissolved ions already in the laser-induced plasma. However, they used 100 ns laser pulse width, we used only $10 \mathrm{~ns}$, and it cannot be excluded that ions are present inside the bubble because of solution vaporization at the plasma edge during its lifetime. Trapping of the early nanoparticles inside the confinement of the cavitation bubble had been observed first by means of timeresolved scanning small-angle X-ray scattering (SAXS) [13]. In the last years, a trend towards imaging of the entire bubble cross section by optical $[14,15]$ and in situ X-ray methods was followed. In this context X-ray radiography and multi-contrast imaging $[16,17]$ was introduced as a complement to in situ SAXS $[18,19]$. Thereby, the distribution of nanoparticles and the ejection time of nanoparticles from the gaseous phase into the surrounding liquid were determined exploiting X-ray absorption contrast [18].

Alternative to anions, organic molecules can be employed for size quenching in situ [20-22], in particular, if the ligand's functional group has high affinity to the inorganic nanoparticle surface [23-25]. But less is known on the size quenching mechanism with macromolecules having no specific anchor group. 
In this work, we address the controversy of the actual temporal and spatial distribution of LAL nanoparticles in the presence of macromolecular ligands to interact during in situ conjugation. This question was addressed by other groups by adding ligands like sodium dodecyl sulfate (SDS) [26] or 2-[2-(2-methoxyethoxy)ethoxy] acetic acid (MEEAA) [27] to the water phase. While these studies provides indication for particle-ligand interaction during an early stage, the size quenching cannot be solely attributed to the presence of the organic backbone as both SDS and MEEAA introduce additional electrolytes or change the $\mathrm{pH}$ of the liquid [5]. Steric stabilization and size reduction by polymers added to the liquid prior to laser ablation was observed for ,e.g., aluminum nanoparticles prepared in poly(vinyl alcohol) (PVA) solution [28], gold nanoparticles in poly(ethylene oxide) (PEG) solution [20] and silver nanoparticles in polyvinylpyrrolidone (PVP) solution [29]. The present study focuses on the functionalization of LAL nanoparticles by PVP. Tsuji et al. have demonstrated that PVP acts as a size quenching ligand in LAL using silver as a target material [29]. In chemical reduction synthesis of silver and gold nanoparticles PVP acts as a shape directing agent in some formulations [30,31] and the same is true for materials like palladium and tellurium [32]. Yet the chemical synthesis of gold nanoparticles with PVP is reported frequently, while spherical morphologies are found [33-36]. Requejo et al. have even reported a route in which PVP leads to spherical gold particles and the omission of PVP results in gold nanorods [37]. Non-spherical nanoparticles were also found during laser processing of nanoparticles when using a $355 \mathrm{~nm}$ laser source and performing laser fragmentation [38], these parameters and the setup are, however, not completely transferrable to those applied in this work.

As a matter of fact, it is possible to control the size of gold nanoparticles to some extent by a controlled delayed addition of oligonucleotides to the colloidal solution $[39,40]$. This indicates 
that quenching by organic ligands can occur ex situ, i.e. outside of the cavitation bubble, by suppressing growth processes like Ostwald ripening and coagulation by steric stabilization of the nanoparticles [41]. Accordingly, there is indication for both possible quenching mechanisms, inside and outside of the cavitation bubble, occurring during LAL in the presence of macromolecular ligands. Therefore, it is mandatory to study nanoparticle formation during an early phase as well, when particles are still trapped inside the cavitation bubble. Due to the complexity of the problem a complementary approach to our earlier in situ SAXS studies is chosen [7, 13, 18, 42]. X-ray Hartmann mask imaging (XHI) was utilized to access multicontrast X-ray imaging, including scattering contrast $[43,44]$. As described earlier in literature $[43,45-$ 47] Hartmann-mask imaging exploits a laterally structured X-ray beam, whose attenuation, pattern shift and structure broadening allows to decompose absorption, differential phase and scattering contrast on a 2D detector. Here, the Hartmann mask consists of a platinum foil with a 2D periodic pattern of holes as shown in Figure 1a-d. The sensitivity maximum of targeted size intervals can be set by variation of the mask pitch and the sample to detector distance [45]. We have chosen a XHI setup with sensitivity maximum at $55 \mathrm{~nm}$ structure size. Hence, secondary particles like large spherical nanoparticles and agglomerates are preferably detected over smaller (primary) particles.

\section{EXPERIMENTAL SECTION}

The experimental details of the liquid-flow in situ LAL setup are described elsewhere in more detail $[7,19,42]$. In brief, laser ablation for in situ experiments was performed by focusing a nanosecond laser (Continuum Minilite I, $1064 \mathrm{~nm}, 7 \mathrm{~ns}, 10 \mathrm{~Hz}$ ) with an average pulse energy of 
$11 \mathrm{~mJ}$ onto the target [19]. The effective fluence, taking the attenuation of the liquid layer at the laser wavelength into account [48], was $33 \mathrm{~J} / \mathrm{cm}^{2}$. A gold ribbon (99.9 \%) having a width of $4 \mathrm{~mm}$ and a thickness of $200 \mu \mathrm{m}$ was used as a target material and continuously transported upwards through the chamber in the same direction as the liquid flow. Ablation was performed in a liquid flow configuration to remove residual nanoparticles for subsequent laser shots. Doublydistilled water solutions of $\mathrm{NaCl}$ and PVP (Acros Organics, 3,500 g/mol, K12, Lot: A0368382) were prepared to a final concentration of $0.5 \mathrm{mM}$. Ex situ LAL was performed using a liquidflow ablation chamber with a liquid height of $5 \mathrm{~mm}$ above the target. A fixed gold plate of $0.5 \mathrm{~mm}$ thickness was used. Ex situ experiments were additionally conducted in Nvinylpyrrolidone (NVP), the monomer of PVP. The chemical was obtained from Acros Organics (99\%, stabilized with $\mathrm{NaOH})$ and used without further purification. Measurements of the $\mathrm{pH}$ value of $1 \mu \mathrm{M}$ and $1000 \mu \mathrm{M}$ solutions of NVP revealed that it was almost constant from 5.9 at 1 $\mu \mathrm{M}$ and 5.9-6.0 at $1000 \mu \mathrm{M}$. Furthermore, the electrolytic conductivity was constant at 0.7-0.8 $\mu \mathrm{S} / \mathrm{cm}$. The liquid volume flow was $50 \mathrm{ml} / \mathrm{min}$, which is three times lower compared to the in situ experiments. For ex situ experiments Milli-Q water was used $(18.2 \mathrm{M} \Omega / \mathrm{cm})$. The laser (Rofin Sinar RS-Marker 100D, $1064 \mathrm{~nm}, 10 \mathrm{~ns}$ ) was operated at $5 \mathrm{~mJ}$ pulse energy and $3 \mathrm{kHz}$ and was scanned across the target surface in a spiral pattern using a galvanometric scanner. The effective fluence was $27 \mathrm{~J} / \mathrm{cm}^{2}$ which is $18 \%$ less as compared to the in situ ablation experiments. To prove that this slight deviation does not affect the particle size distributions significantly additional size distributions obtained by ex situ LAL using an effective fluence of $36 \mathrm{~J} / \mathrm{cm}^{2}$ are compared with those obtained at $27 \mathrm{~J} / \mathrm{cm}^{2}$ in Figure S1. The ablation chamber was made out of polytetrafluorethylene (PFTE) to avoid contamination of the product colloids by ions that might otherwise be dissolved of chambers made of e.g., steel or aluminum. For 
transmission electron microscopy (TEM) (Zeiss EM 910, $120 \mathrm{kV}$ ) the colloids were drop-casted onto a carbon-coated copper grid. Automatic image analysis was performed by the ImageJ (Version 1.51s) plugin ParticleSizer [49]. Analytical disc centrifugation (ADC, CPS instruments) was performed in a sugar density gradient and constantly operated at maximum speed of $24,000 \mathrm{rpm}$. Gold nanoparticles of $6 \mathrm{~nm}$ hydrodynamic diameter were used as calibration standard. In ADC the integral intensity of the entire injected sample mass is calculated by Mie theory and from the derived cumulative surface-weighted size distribution the specific surface area is accessible. Hence, for each data point the additive concentration per nanoparticle surface area or mass in $\mu \mathrm{mol} / \mathrm{cm}^{2}$ or $\mu \mathrm{mol} / \mathrm{mg}$ is derived, respectively. Note that the specific surface area and the median diameter are mutually linked quantities. Still, the specific surface area integrates every size fraction within the size distribution of the gold colloids as every particle contributes to the overall specific surface area. UV/Vis extinction spectroscopy was performed using a spectrophotometer (Thermo Scientific Evolution 201). Spectroscopy was used to calculate the ablated gold mass from the concentration of the ex situ synthesized colloids. Representative spectra from gold nanoparticles produced in pure water, $0.5 \mathrm{mM} \mathrm{NaCl}$ and PVP solutions and the calibration of the UV/Vis spectra by gravimetrical analysis of the ablated gold mass are given in Figure S2. Viscosities were measured using a rheometer (Anton Paar, Physica MCR 301).

Time-resolved visible-light shadowgraph of the cavitation bubbles in water and PVP solution were performed in a self-designed 3D-printed ablation chamber made of acrylonitrile butadiene styrene. A liquid flow rate of $35 \mathrm{ml} / \mathrm{min}$ was applied to remove residual nanoparticles and persistent bubbles between two laser pulses. The laser repetition rate was set to $0.2 \mathrm{~Hz}$. Additional information on the used shadowgrapy setup is presented in the Supporting Information, including the recorded image sequences (Fig. S3-S6). 
The platinum-based Hartmann mask used for multi-contrast X-ray imaging consists of a $25 \mu \mathrm{m}$ thick foil (Chempur, 99.9\%) with regular holes of about $10 \mu \mathrm{m}$ in diameter, drilled by pulsed fslaser micromachining (Spectra Physics Spitfire Ace, $800 \mathrm{~nm}, 100 \mathrm{fs}$ ) using a fluence of $2 \mathrm{~J} / \mathrm{cm}^{2}$ at a repetition rate of $5 \mathrm{kHz}$. The holes cover the rectangular pattern $(7 \times 5 \mathrm{~mm})$ with a pitch of $65 \mu \mathrm{m}$. Micrographs of the Hartmann mask are shown in Figure 1 from the front and back side. Light microscopy allows to visualize the funnel shape of the holes on the front side, perpendicular to the incident transmitted fs-laser (Fig. 1a,b). The back side of the mask does not exhibit any funnels but a regular pattern of micron sized holes (Fig. 1c,d). A scheme depicting the basics of the exploited imaging technique for in situ measurements during LAL is given in Figure 1e. Absorption of X-rays occurs whenever a beamlet, shaped by the shadow mask, interacts with matter e.g., after transmission through water. Transmission increases at the occurrence of the cavitation bubble in LAL with respect to the surrounding liquid.

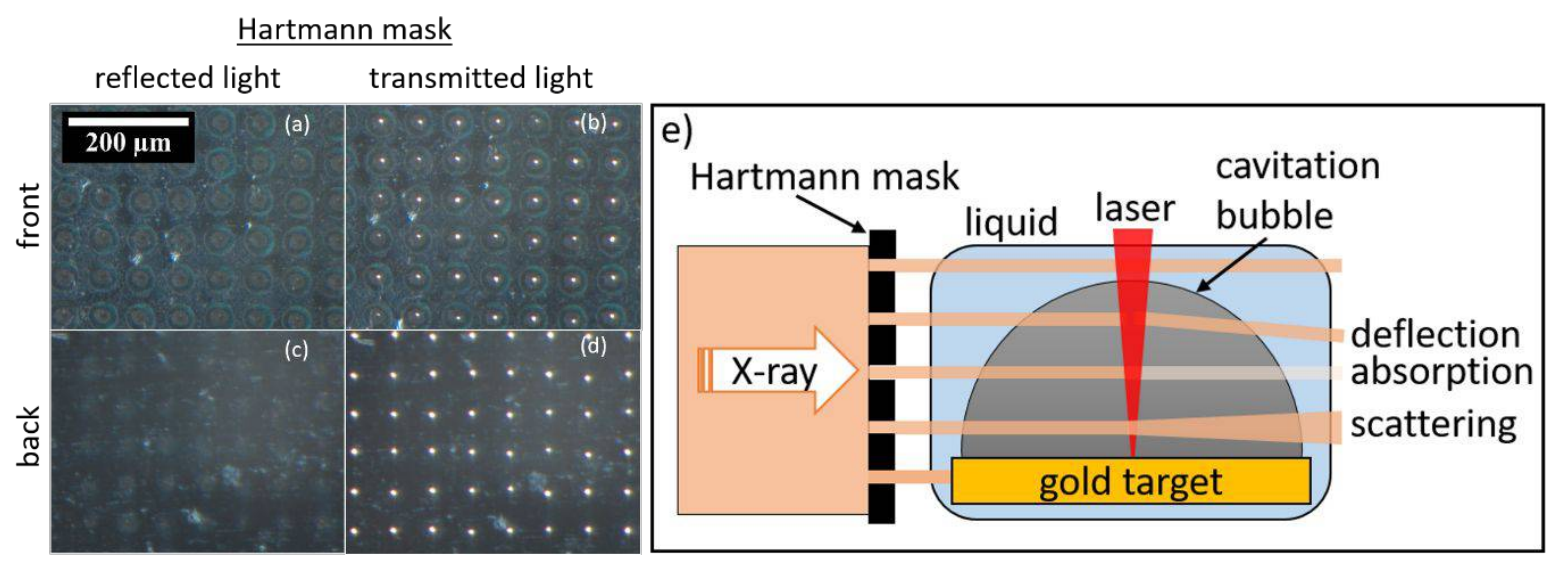

Figure 1. Images of the Pt foil acting as a Hartmann mask for in situ experiments obtained by optical microscopy. Front of the mask in reflected (a) and transmitted light mode (b). The laserdrilled holes are funnel-shaped on the front side (side at which the laser beam impinges). Back of the mask in reflected (c) and transmitted light mode (d). Sketch of the XHI approach with the incoming X-ray beam being split into multiple beamlets by the Hartmann mask, and undergoing 
absorption, differential phase shift (deflection) and diffuse scattering (broadening) when crossing the cavitation bubble and the confined nanoparticles (e).

XHI experiments were performed at the ID19 beamline of the European Synchrotron Radiation Facility (ESRF, Grenoble, France) using a quasi-monochromatic beam from a single-line undulator with an energy of $17.9 \mathrm{keV}$. The X-ray beam was restricted to an area of $7 \mathrm{x} 5 \mathrm{~mm}$ by slits to illuminate only the Hartmann mask. The array of beamlets formed by the Hartmann mask passes the sample cell of $5 \mathrm{~mm}$ water to be converted to visible light by a YAG scintillator of $100 \mu \mathrm{m}$ thickness. The distance between the ablation point and the scintillator was $30 \mathrm{~mm}$. A fast CMOS camera (PCO dimax) recorded the intensity distribution with $40 \mu$ s exposure time, a sequence of images around the ablation event spaced $100 \mu$ s was taken. An interleaving of several films with shifted delay time between acquisition and the laser impact allowed to construct a movie with $40 \mathrm{kHz}$ (25 $\mu$ s spacing) image rate. A repetition of the image acquisition for a total of at least 3,000 laser shots was performed for every liquid in order to increase the signal-to-noise ratio. XHI is a multi-contrast X-ray imaging technique. Details on image formation and reconstruction are described in detail in $[16,43,44,50,51]$. Therefore, here only a short description shall be given. By usage of a Hartmann mask the intensity, position and broadening of the beamlets reveals three different contrast modes (absorption, differential phase, and scattering, respectively). In this publication the scattering contrast is of central interest as this stems from nanoscale inhomogeneities. The image reconstruction was performed identically to a previous publication by Reich et al. [51]. Instead of individual beamlet analysis a Fourier transform of the beamlet pattern allows to separate the different contrasts. Therefore, the Fourier transform of the pattern is followed by a back transform of the specific harmonics of the Fourier spectrum. Particularly, the 
cavitation bubble creates absorption contrast, which imprints on the scattering channel [50]. As reported earlier, this crosstalk from the absorption contrast to the scattering contrast can be removed by a decorrelation procedure $[51,52]$.

\section{RESULTS AND DISCUSSION}

The goal of the present study is to clarify the mechanism of nanoparticle-macromolecular ligand interaction during LAL. Here, we use (i) pure water as a negative (null effect) reference liquid, (ii) $\mathrm{NaCl}$ (aq.) as positive reference. $\mathrm{NaCl}$ has the ability to quench the size of nanoparticles already inside the gaseous phase of the cavitation bubble, as shown before by scanning SAXS [7]. The additive (iii) that is employed is the macromolecular ligand PVP. It is utilized in the exact same molar concentration as $\mathrm{NaCl}$ to ensure direct comparability of the molar size quenching efficiencies. As PVP does not contain mercapto groups (-SH), no covalent bond (chemisorption) to the gold nanoparticle surface is expected. Yet, the $\mathrm{N}-\mathrm{C}=\mathrm{O}$ groups of the PVP backbone bind to the gold surface by physisorption [53-55]. Although PVP is frequently used as a stabilizing ligand for silver [29, 30, 56] rather than gold [35] nanoparticles, a gold target is used here. This is motivated by gold nanoparticles proving to be effectively quenched by $\mathrm{NaCl}$ [7]. It is shown that $\mathrm{NaCl}$ exhibits the ability to quench the size of gold nanoparticles already inside the cavitation bubble (i.e. in situ).

Using absorption contrast, it is possible to record the cavitation bubble as shown in the inset of Figure $2 \mathrm{~b}$. Scattering contrast is a result of diffuse scattering of X-rays and originates from scattering at inhomogeneities of the probed specimen [45]. The angular width of scattering scales inversely to the object size according to the principles of small-angle X-ray scattering [46, 57]. 
In the present setup the sensitivity ranges from $20-230 \mathrm{~nm}$ in sizes (at 50\%, with a maximum at $55 \mathrm{~nm}$ ). The calculated sensitivity curve is shown in Figure 2a, which was calculated from our setup parameters according to the equation derived by Lynch et al. (equation (68)) [45]. The detection of small primary particles $(\mathrm{d}<15 \mathrm{~nm})$ by using XHI is negligible, and primary particle size shifts by the addition of $\mathrm{NaCl}$ are also not detected by SAXS [7] because SAXS is limited in differentiation of overlapping size fractions [58]. Hence, for the scope of the present study, the secondary size fraction is the main region of interest, as it is known to be connected to the product colloids' bimodality. The shifted sensitivity towards particles even larger than $100 \mathrm{~nm}$ adds complementary information on earlier works. In general, imaging offers prospects to map spatial distributions as opposed to localized SAXS. Within our sensitivity limit we see a homogenous scattering distribution across the bubble. At the same time size information in the classical sense in XHI is lost, therefore size quenching (of large particles) is translated to intensity changes. Anyway, also in situ SAXS does not reveal changes in the size of small primary particles induces by $\mathrm{NaCl}$ inside the cavitation bubble (Fig. S7). 

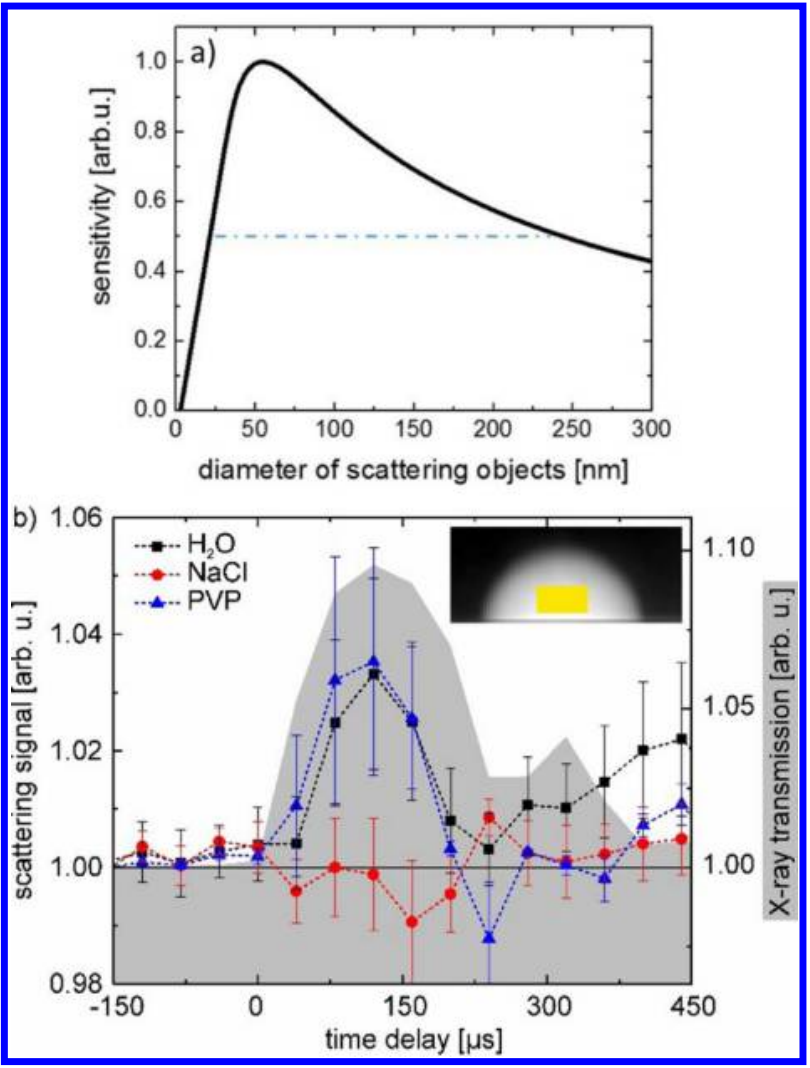

Figure 2. Scattering sensitivity on nanoparticles as function of the scattering object size for the given setup. The blue dashed-dotted line indicates the size interval above $50 \%$ relative sensitivity (a). Scattering signal as function of the delay after laser impact obtained at the center of the cavitation bubble (inset shows the transmission signal together with the region of interest) (b). Scattering signals of gold nanoparticles in pure water (black squares), $\mathrm{NaCl}$ - (red circles) and PVP-solution (blue triangle) (both $0.5 \mathrm{mM}$ ). The shaded curve represents the change in X-ray transmission starting from 1 ( $0 \%$ intensity change) before laser arrival. Error bars are the standard deviation between several runs.

By using negative delays (before laser impact) for the flat field correction, it is possible to remove signal from residual nanoparticles inside the ablation chamber volume that may not transported off by the water flow. As visible from the black squares in Figure $2 b$ the scattering 
signal of gold nanoparticles as ablated in pure water observed at the center of the cavitation bubble (inset) follows the growth and shrinking of the first and second cavitation bubble, the latter frequently referred to as bubble rebound. The relation of the cavitation bubble and the confined particles ablated in pure water further confirms (i) that the observed secondary nanoparticles are confined within the gaseous phase of the cavitation bubble and (ii) are retracted towards the target surface upon bubble shrinkage. Therefore, the first reference measurement of gold nanoparticles in pure water proves the validity of XHI for nanoparticle detection during LAL by reproducing the bubble-nanoparticle SAXS-behavior which is known from the literature $[7,13,42]$.

The second reference experiment is the laser ablation of gold nanoparticles in a $0.5 \mathrm{mM} \mathrm{NaCl}$ solution. The temporal distribution is given by the red circles in Figure $2 \mathrm{~b}$ representing the scattering signal from the nanoparticles. The scattering signal of nanoparticles ablated in a micromolar electrolyte solution is, at almost any delay after laser impact, as low as for the reference measurements at negative delays. It was shown before by means of in situ SAXS that the size and the amount of secondary particles is reduced inside the cavitation bubble already $90 \mu \mathrm{s}$ after laser impact [7]. As XHI is mostly sensitive to secondary particles (Fig. 2a) it is concluded that the size reduction decreases the scattering signal intensity below the detection limit. Consequently, less to no signal is recorded.

Just like the $\mathrm{NaCl}$ solution the PVP solution is prepared prior to LAL so that PVP molecules are present in the liquid phase at any time during the ablation process. The blue triangles in Figure $2 \mathrm{~b}$ show the scattering signal of nanoparticles ablated in presence of PVP as a function of the time delay after laser impact. Obviously, the curve progression is similar to the one in pure water. The results obtained from direct measurement of the particle X-ray scattering in situ (i.e. 
inside the cavitation bubble) show that the macromolecular ligand PVP does not affect the abundance of large gold nanoparticles (or aggregates) within the first $210 \mu$ s after laser impact. This result is somewhat surprising, as it has been shown by several groups that size quenching of nanoparticles synthesized by LAL is possible by adding macromolecular ligands to the liquid phase before performing LAL [20, 26, 59]. Still, none of the references include direct in situ observation of the processes inside the gaseous phase of the cavitation bubble. Our data indicates the ineffectiveness of macromolecular ligand functionalization during cavitation bubble confinement.

Regarding the in situ XHI measurements it is concluded that PVP does not act as a size quenching agent for the secondary particles on the observed time scale during cavitation. This might be due to slow reaction kinetics, low diffusion constant or by the partial disintegration of PVP molecules by the harsh conditions induced by the laser impact. In principle, also a too low concentration could cause the observed ineffectiveness of PVP. This is excluded in the following section, addressing the ex situ analysis of size quenched nanoparticles. The size quenching efficiency of micromolar solutions of $\mathrm{NaCl}$ has been proven before in situ and ex situ. In contrast to $\mathrm{NaCl}$, the macromolecular ligand PVP is less frequently used in combination with gold nanoparticle for size control during LAL. It is therefore mandatory to prove the size quenching ability of PVP with well-established ex situ methods to support the results from the in situ XHI. As the scattering contrast of the XHI method is based on scattering of X-rays it is sensitive to particle volume (mass). We will therefore compare the volume-weighted particle sizes when comparing the ex situ to the in situ results. To discriminate the influence of PVP on the size of the ablated gold nanoparticles, either by chemical functionality or molecular size, additional ablation in N-vinylpyrrolidone (NVP), which is the monomer of PVP, was performed. 
The volume-weighted median diameters $\mathrm{D}_{50}$ of gold nanoparticle synthesized in $\mathrm{NaCl}$, PVP and NVP are plotted in Figure $3 \mathrm{a}$ as a function of the additive-to-gold-ratio (AGR). These median diameters are derived from ADC while the ablated gold mass is calculated from the UV/Vis extinction spectra of the gold colloids. The two quantities allow for the calculation of the specific surface area. The absolute additive concentrations are varied between $1 \mu \mathrm{M}$ and $1,000 \mu \mathrm{M}$. As shown in Figure 3a using solutions of $\mathrm{NaCl}$ with a concentration as low as $0.2 \mu \mathrm{mol} / \mathrm{mg}$ is sufficient to start size quenching and a size reduction from $40 \mathrm{~nm}$ (pure water) to $12 \mathrm{~nm}$ in the volume-weighted average. As colloids produced in $1 \mu \mathrm{M}(0.02 \mu \mathrm{mol} / \mathrm{mg})$ solutions of $\mathrm{NaCl}$ were unstable after minutes to hours independent of the AGR these results are excluded.

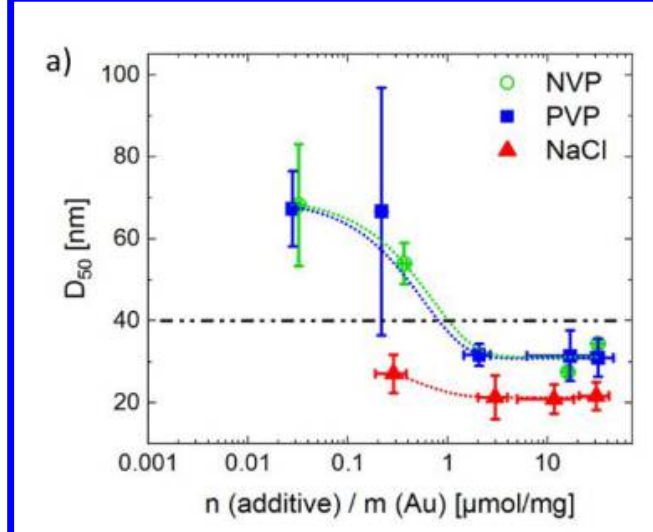

c)
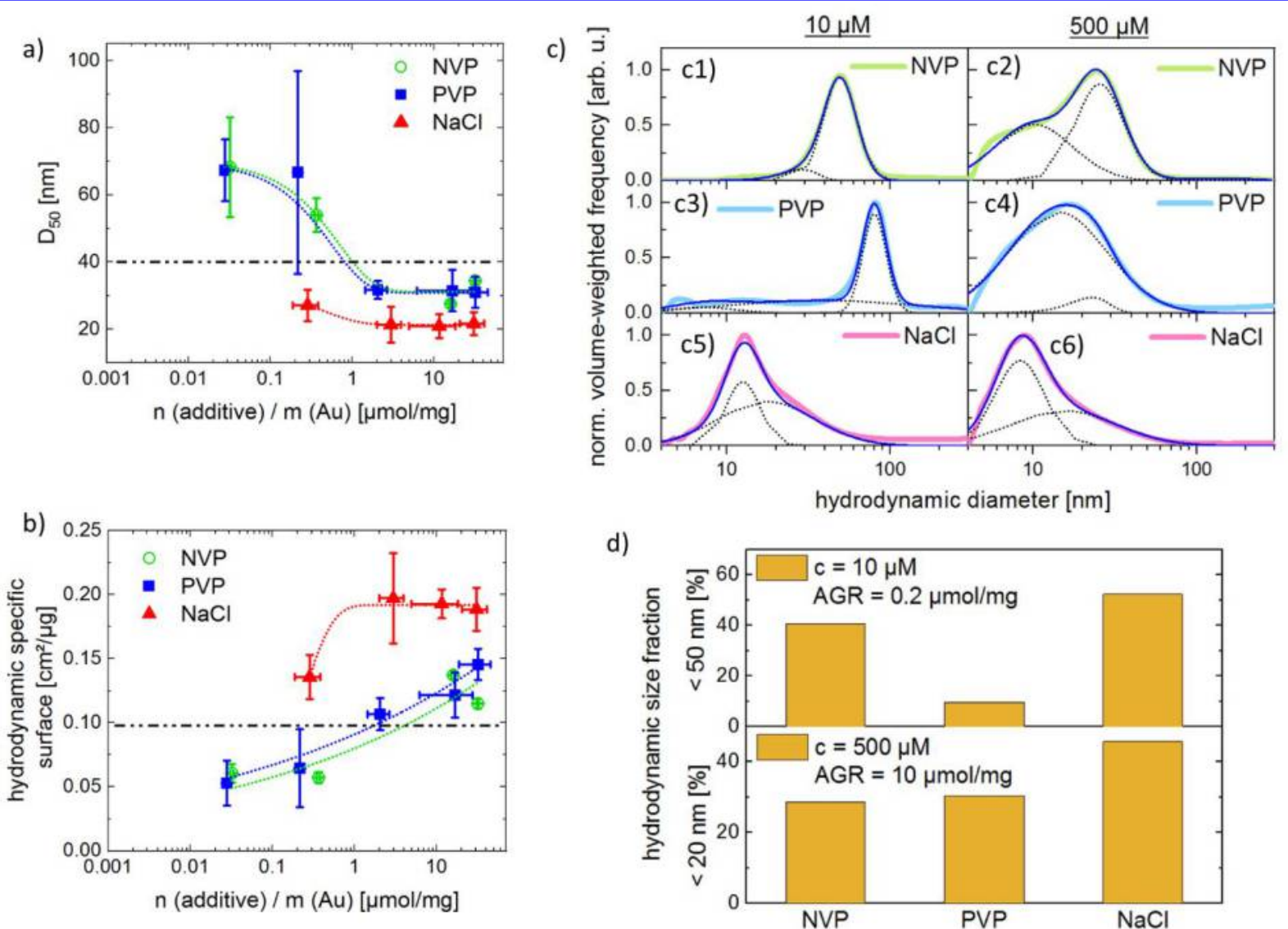

d)

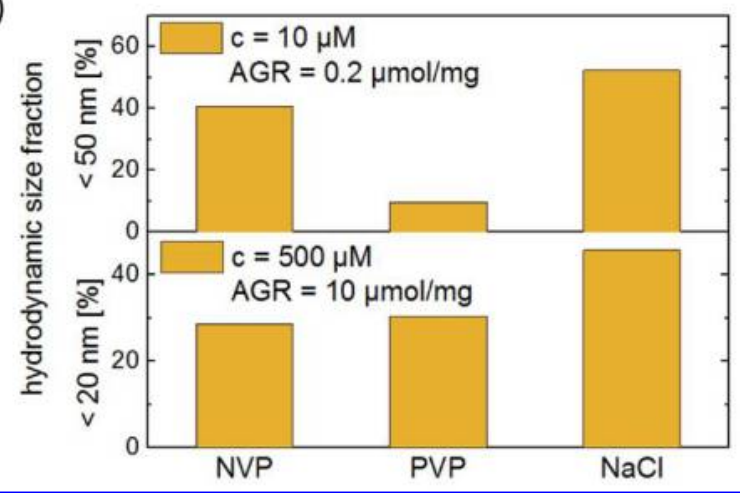

Figure 3. (a) Volume-weighted hydrodynamic median diameter obtained by analytical disc centrifugation and (b) related specific surface area of gold nanoparticles in PVP and $\mathrm{NaCl}$ as 
function of the additive-to-gold ratio (AGR). The dashed-dotted horizontal lines mark the reference experiments from LAL in pure water. Nanoparticle mass concentrations are obtained by optical UV/Vis extinction spectroscopy. (c) Volume-weighted particle size distributions of colloids in low and high additive concentrations derived from ADC analysis. (d) Hydrodynamic size fractions of particles $<20 \mathrm{~nm}$ and $<50 \mathrm{~nm}$. The data is derived from the ADC size distributions in (c). The results display the properties of the particles in their nascent hydrodynamic environment.

The function is saturated already at AGR of $2 \mu \mathrm{mol} / \mathrm{mg}$ resulting in hydrodynamic nanoparticle diameters of $20 \mathrm{~nm}$. As these values are volume-weighted they prove the absence of considerable amounts of large secondary nanoparticles or aggregates. This finding corresponds well with the absence of in situ XHI scattering signal for $\mathrm{NaCl}$ in Figure 2b. PVP is suitable for size control of laser generated gold nanoparticles, if high concentrations are used. Still, the trend differs significantly from $\mathrm{NaCl}$. While the relative concentration at saturation is similar for $\mathrm{NaCl}$ and PVP (1-2 $\mu \mathrm{mol} / \mathrm{mg}$ ) the size reducing effect is lower for PVP (minimum size only $30 \mathrm{~nm}$ ). At low concentrations $(<0.5 \mu \mathrm{m} / \mathrm{mg})$ even a destabilization of particles seems to take place. The specific surface dose of additives per nanoparticle surface is plotted in Figure $3 b$. The ordinate shows the specific surface derived from $\mathrm{ADC}$. The electrolyte $\mathrm{NaCl}$ shows a step-like behavior as a function of the AGR with a saturation at $2 \mu \mathrm{mol} / \mathrm{mg}$. Regarding PVP, this plot shows that the specific surface area is increasing as a function of the AGR while no saturation is reached within the investigated range (Fig. 3b). In sum, the volume-weighted and surface related dependencies derived from the hydrodynamic analysis indicate a size quenching efficiency that is expected to correlate to the in situ XHI measurements (Fig. 2b) when the amount of PVP per 
gold mass exceeds $\sim 10 \mu \mathrm{mol} / \mathrm{mg}$. Note that the absolute concentration of PVP in XHI of $500 \mu \mathrm{M}$ (equivalent to $\sim 10 \mu \mathrm{mol} / \mathrm{mg}$ ex situ) is even higher in terms of AGR, but difficult to quantify in the dynamic environment due to the unknown time-averaged ablation yield. Apart from that, an effect of the PVP concentration on the laser transmission and LAL-cavitation can be excluded by measuring the extinction spectrum of the pure solution and bubble dynamics (as discussed later). It is concluded that PVP mainly acts as a growth quencher ex situ and that the effect is therefore not observable in situ. Note that the data in Figure 3 display the hydrodynamic sizes of the colloids. Keeping this in mind it is obvious that the large particles observed in PVP solutions with AGR $\leq 0.1 \mu \mathrm{mol} / \mathrm{mg}$ are not individual nanoparticles but rather large agglomerates (Fig. S11). These are formed, just as in pure water, by insufficient repulsive forces between the individual nanoparticles. While PVP binds to the gold surface the respective concentrations are just too low to induce steric stabilization of the many small particles because the overall surface in the system is high if the number of small particles is high as well. Therefore, the amount of PVP is just enough to stabilize the reduced (total) surface of the forming agglomerates. This behavior of agglomerate stabilization supports the conclusion that PVP acts as an ex situ growth quencher rater that already in situ inside the cavitation bubble.

To clarify the influence of the organic ligand PVP in terms of the size of the molecules and their functionality we performed additional ex situ experiments in different concentrations of solutions of the PVP's monomer N-vinylpyrrolidone (NVP). The chemical was obtained from Acros Organics ( $99 \%$, stabilized with $\mathrm{NaOH})$ and used without further purification. Measurements of the $\mathrm{pH}$ value of $1 \mu \mathrm{M}$ and $1000 \mu \mathrm{M}$ solutions of NVP revealed that it was almost unaffected, with 5.9 at $1 \mu \mathrm{M}$ and 5.9-6.0 at $1000 \mu \mathrm{M}$. Furthermore, the electrolytic conductivity was constant at $0.7-0.8 \mu \mathrm{S} / \mathrm{cm}$. The volume-weighted median diameter and the specific surface area 
of the gold nanoparticles was derived from combined ADC and UV/Vis analysis. The graphs in the Figures 3a,b reveal an almost identical curve progression of samples produced in NVP compared with those obtained in PVP but quite different to $\mathrm{NaCl}$. This result points toward a major influence of the additives' functionality rather than the size of the molecule. Hence, NVP and PVP of the same molarity have quite similar effect on the final size of the gold nanoparticles although NVP's molecular weight is only 1/35 of PVP (and roughly twice as large as that of $\mathrm{NaCl}$ ). Both organic additives, NVP and PVP, do not bear functional groups capable to chemisorb on the gold surface obviously affect the size of the nanoparticles on a later time scale, probably after the final collapse of the cavitation bubble.

The similarity of the curve progression of NVP and PVP samples in Figure 3a,b requires further clarification. Therefore, in Figure 3c the size distributions (ADC, volume-weighted) of low and high concentrations of NVP and PVP are compared. Additional surface-weighted size distributions are presented in Figure S8. While at concentrations of $1 \mu \mathrm{M}$ the size distributions are quite similar, at $1000 \mu \mathrm{M}$ the entire PVP distribution is shifted to smaller hydrodynamic particle sizes whereas the NVP distribution shows only the evolution of a weaker primary mode at small particle sizes. Both additives are less effective in terms of their size quenching efficiency compared to $\mathrm{NaCl}$ (red line). The reason why the distinct differences in the size distributions of NVP and PVP are not displayed in Figure 3 are that (i) the median diameter does not describe all details of a size distribution and (i) there are variations of abundance of particles larger than 100 nm in NVP and PVP (Fig. 3c). Accordingly, while organic ligand's the functional group seems more important to size quenching compared to the molecular weight, the latter influence is not negligible with regard to the hydrodynamic diameter. The reduced abundance of hydrodynamically large $(>20 \mathrm{~nm})$ particles in PVP is a proof for steric stabilization which is 
important for the colloid's long term stability against aggregation. This is clarified in Figure $3 \mathrm{~d}$ by plotting the relative hydrodynamic size fraction of particles $<50 \mathrm{~nm}$ and $<20 \mathrm{~nm}$ as a function of the respective additive.

To summarize the hydrodynamic ex situ particle analysis, it is demonstrated that both additives result in smaller nanoparticles. Yet, while the addition of the electrolyte $\mathrm{NaCl}$ results in a plateau of the specific surface area already at a low AGR of $1 \mu \mathrm{mol} / \mathrm{mg}$, the macromolecular ligand does not saturate even at $20 \mu \mathrm{mol} / \mathrm{mg}$. This indicates that $\mathrm{NaCl}$ results in an additional energy barrier against ripening or coalescence of the nanoparticles (or their atom cluster seeds) by increasing the surface charges. On the other hand, PVP stabilizes nanoparticles sterically, building up a short-ranged steric barrier between the particles. With increasing amount of PVP molecules that barrier becomes more bulky and provides quenching of subsequent ripening processes. Hence, the hydrodynamic analysis indicates that the differing colloidal stabilizing mechanisms of $\mathrm{NaCl}$ and PVP also results in different size reductive effects in LAL.

To complement the ex situ particle analysis additional particle characterization by TEM has been performed. This is to provide additional information compared to a sole hydrodynamic analysis, as TEM is sensitive to individual nanoparticles. The results are collected in Figure 4 (with TEM pictures shown in the Supporting Information, Fig. S9-S12). The left column (black bars) shows the number-weighted histograms of gold nanoparticles ablated in pure water, low $(0.2 \mu \mathrm{mol} / \mathrm{mg}$, corresponds to $10 \mu \mathrm{M})$ and high $(10 \mu \mathrm{mol} / \mathrm{mg}$, corresponds to $500 \mu \mathrm{M})$ AGRs of $\mathrm{NaCl}$ and PVP. It is visible that both additives reduce the size of the individual, numberweighted, nanoparticles with respect to pure water from $19 \mathrm{~nm}$ down to 6-8 $\mathrm{nm}$ with increasing AGR. As visible from the number-weighted TEM histogram in the left column of Figure 4, the nanoparticles synthesized in pure water exhibit a distinct, broad, and bimodal size distribution 
with an average size of $17 \mathrm{~nm}$ and a significant share of large solid spheres of $40-70 \mathrm{~nm}$ in diameter. If $\mathrm{NaCl}$ is used in a small AGR of $0.2 \mu \mathrm{mol} / \mathrm{mg}$ the small particles (size reduction by $2 \mathrm{~nm}$ ) are less affected than the large solid spheres. The size of the latter (maximum diameter $40 \mathrm{~nm}$ ) and relative amount decrease drastically. This effect is enhanced by LAL with a high AGR of $10 \mu \mathrm{mol} / \mathrm{mg}$, which reduces the maximum size of large solid spheres to $20 \mathrm{~nm}$ and halves the size of the primary particles. Regarding LAL in PVP the situation is slightly different, especially at high AGR. Even an AGR of $10 \mu \mathrm{mol} / \mathrm{mg}$ of PVP leaves a significant share of $40 \mathrm{~nm}$-particles behind. Regarding the number-weighted-histogram of gold nanoparticles in high AGR of NVP, the similarity to the size distribution in PVP is obvious. Still, the distribution is broader in NVP, while in PVP a mode at diameters as small as $6 \mathrm{~nm}$ is dominant which is accompanied by a shoulder in the distribution. For reasons of comparability to the in situ XHI analysis the histograms are also converted from number- to volume-weighted distributions. By this statistics large particles are amplified. The corresponding histograms (Fig. 4, middle column, red bars) show that $\mathrm{NaCl}$ indeed reduces the size and abundance of large particles, as the median diameter $\mathrm{D}_{50}$ is significantly reduced from $52 \mathrm{~nm}$ in pure water down to $10 \mathrm{~nm}$ at $10 \mu \mathrm{mol} / \mathrm{mg}$ AGR. 


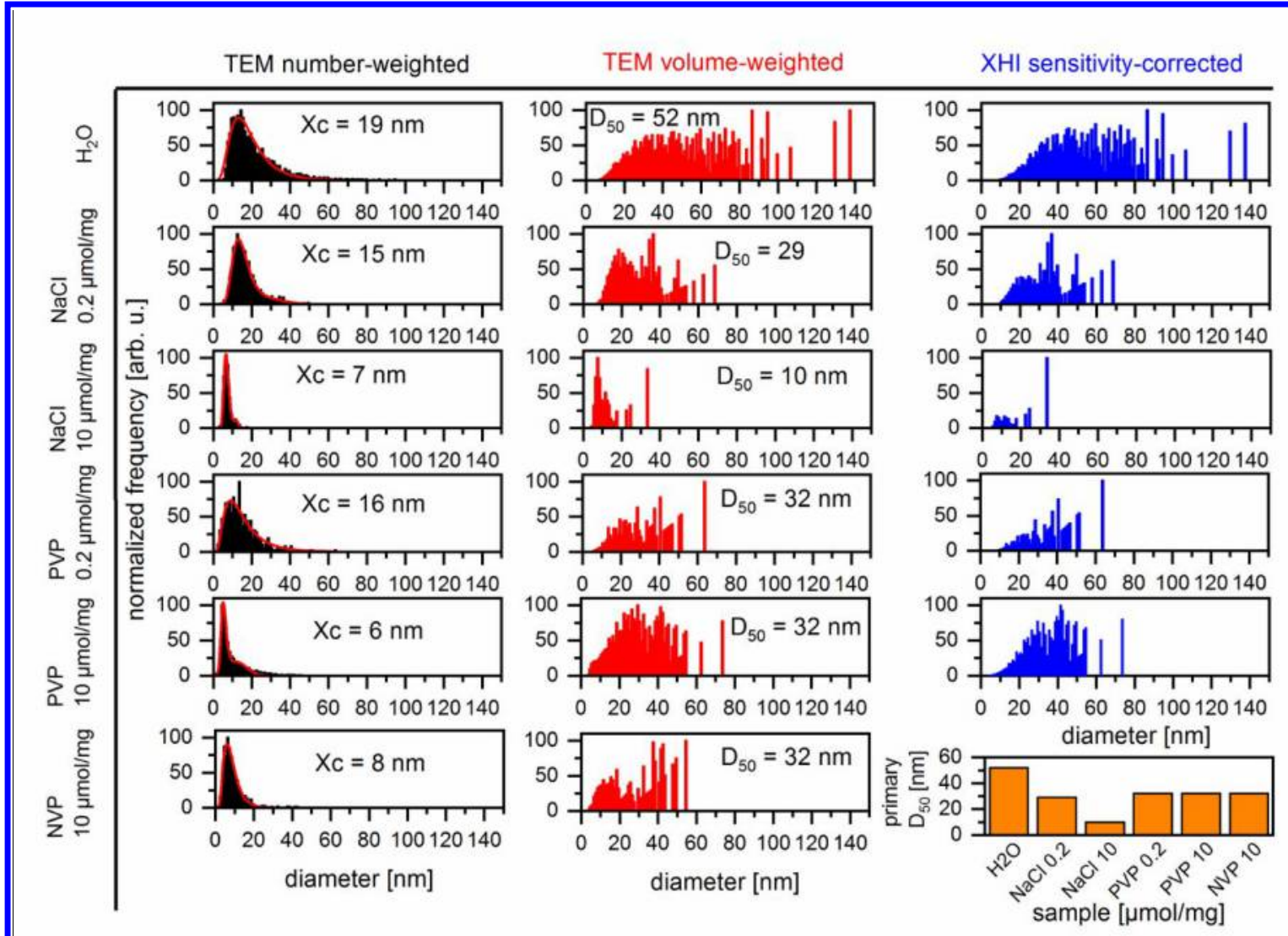

Figure 4. Representative TEM histograms of AuNP in pure water, $\mathrm{NaCl}$ and PVP at AGRs of

0.2 and $10 \mu \mathrm{mol} / \mathrm{mg}$ (left column). The envelopes shown as solid red lines are obtained by lognormal fitting and the center of gravity Xc is displayed. The middle column shows the calculated volume-weighted histograms of the same samples with corresponding median diameters $\mathrm{D}_{50}$. In the right column the volume-weighted histograms are further weighted by the XHI sensitivity curve shown in Figure 2a. The plot in the lower right corner sums up the volumeweighted primary particle median diameters as a function of the type and AGR of additive.

However, in the case of the macromolecule PVP the situation is different. While already small amounts of PVP (AGR of $0.2 \mu \mathrm{mol} / \mathrm{mg}$ ) significantly reduce the median particle size from $52 \mathrm{~nm}$ to $32 \mathrm{~nm}$, no further reduction in observed with increasing AGR. The volume-weighted distribution in high AGR of NVP is quite similar to that in low AGR of PVP (the lack of 
particles $<60 \mathrm{~nm}$ is due to the low number of large particles). It is concluded that the monomer and the polymer affect the primary particles in the same way but PVP is much more effective due to its higher chain-length and the resulting improved resistance against hydrodynamic ripening of the nanoparticles. The primary median diameters (volume-weighted) are displayed in Figure 4 (lower right) as a function of additive and concentration. All used additives and concentrations reduce the median particle size by at least $\sim 20 \mathrm{~nm}$. Still, high AGRs of NaCl are the only sample that quenches the particle size even down to $10 \mathrm{~nm}$, which is therefore not detectable inside the cavitation bubble by XHI.

As discussed before the in situ XHI is sensitive to the ablated particle volume, but the overall size-dependent sensitivity is not a linear function. Therefore, the sensitivity curve (Fig. 2a) is applied to the volume-weighted TEM histograms, resulting in histograms mapped onto the XHI sensitivity shown in the right column of Figure 4 (blue bars). Significant differences in the histograms are observable as function of the liquid composition. The sensitivity-weighted histogram of the colloid produced in pure water peaks at $90 \mathrm{~nm}$ and exhibits an overall broad distribution. The finding fits to the in situ XHI analysis (Fig. 2b) as the expected strong scattering signal is indeed observed. Additionally, the high AGR of $\mathrm{NaCl}$, as used for the in situ analysis, shows a scattering sensitivity maximum at about $35 \mathrm{~nm}$, with all other particle species in the size range of $\leq 25 \mathrm{~nm}$. Therefore, assuming that $\mathrm{NaCl}$ already quenches the size of nanoparticles in situ inside the cavitation bubble, no XHI scattering signal is expected. It is therefore plausible that the electrolyte $\mathrm{NaCl}$ not solely reduces aggregation inside the cavitation bubble, but also the size and abundance of individual large nanoparticles. The sensitivityweighted TEM-histogram at $10 \mu \mathrm{mol} / \mathrm{mg}$ AGR PVP peaks at $45 \mathrm{~nm}$ and the scattering signal remains high up to $75 \mathrm{~nm}$, because of the higher sensitivity of the XHI setup towards larger 
particles (Fig. 2a). Again, assuming PVP would affect the size of ablated nanoparticles already inside the cavitation bubble a reduced scattering signal in XHI would result, compared to the ablation in pure water. As this is not the case the conclusion is that PVP does not induce size quenching to individual nanoparticles already inside the cavitation bubble, but acts as a growth and ripening quencher on a later time-scale, thereby reducing the final particle size of both small primary and large secondary particles. 

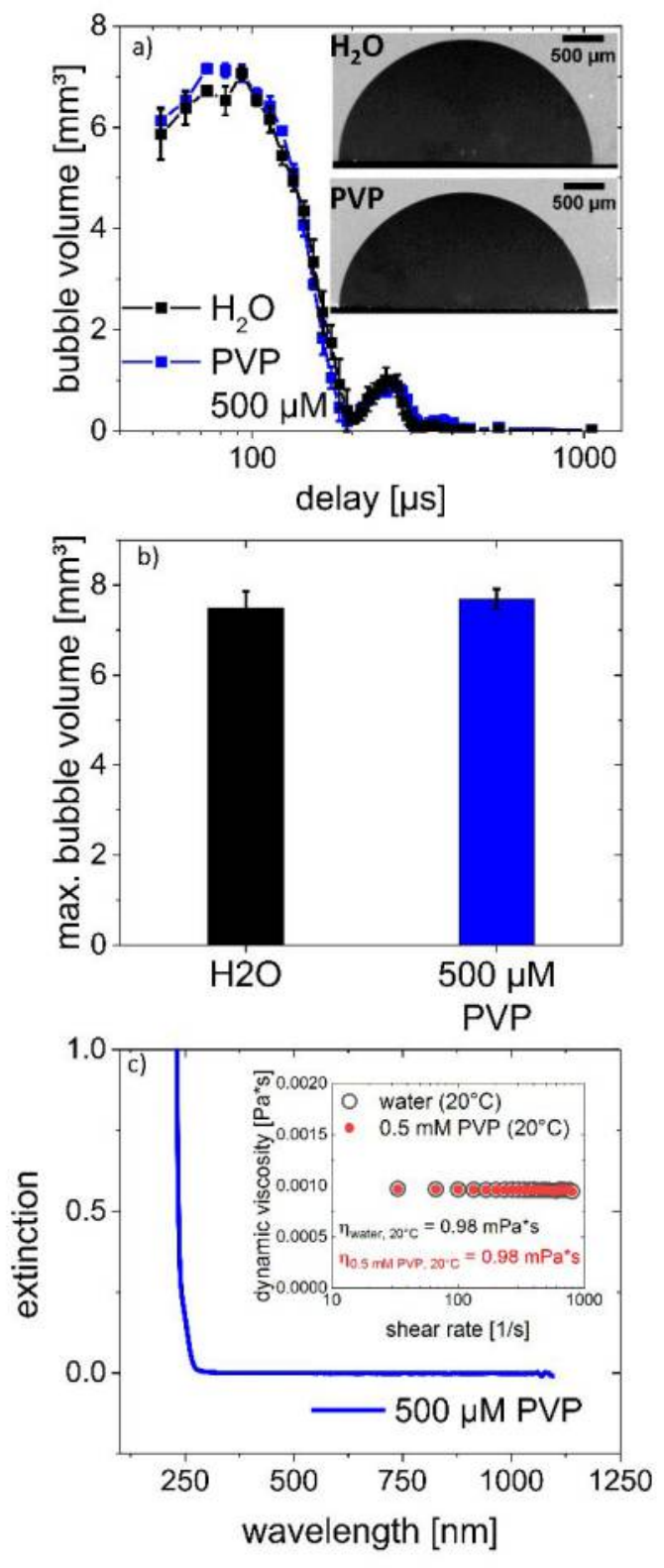

Figure 5. (a) Cavitation bubble dynamics as function of the delay from the laser impact. The PVP molecules do not affect the dynamics of the bubble.(b) Results of image analysis of the cavitation bubble at its maximum extension at $93 \mu$ s after laser impact on the target showing no difference between pure water and $500 \mu \mathrm{M}$ PVP solution. (c) UV/Vis extinction spectrum of a $500 \mu \mathrm{M}$ PVP solution. The solution is transparent at the laser wavelength of $1064 \mathrm{~nm}$. Inset: Dynamic viscosity of pure water and a $0.5 \mathrm{mM}$ PVP solution. 
As both the in situ and the ex situ (hydrodynamic and structural analysis) point towards a growth quenching mechanism of the macromolecular ligand PVP at a time-scale after the lifetime of the cavitation bubble, we need to exclude processes related to the laser ablation and cavitation itself that might cause this behavior. For example, Tsuji et al. have found a size quenching effect of PVP on silver nanoparticles during LAL [29]. They attributed this effect at least partially to the reduced size and lifetime of the laser-induced cavitation bubble, when PVP was used instead of pure water. Still, in our case the situation is different. The molecular weight of the PVP used in the present study is almost three times lower compared to Tsuji et al. [29], additionally the concentration we used for the in situ analysis $(500 \mu \mathrm{M})$ is 36 times lower here . Overall this sums up to a negligible (factor of 108) influence of the PVP on the hydrodynamics. This is verified by performing shadowgraphic imaging of the cavitation bubble produced during LAL of gold in water with $500 \mu \mathrm{M}$ PVP. As shown in Figure $5 \mathrm{~b}$ the maximum bubble volume (93 $\mu$ s after laser impact) is independent of the presence of PVP molecules. Therefore, it is underlined that the effective laser fluence on the gold target surface is the same for both liquids, as it is known that the cavitation bubble volume scales with the laser fluence [19]. The overall dynamics of the cavitation bubble (e.g. the volume and peak time of the bubble rebound) are also not significantly affected by the PVP molecules as shown in Figure 5a. The effective laser fluence on the target surface due to absorption in the solution is not expected to be affected by the low concentration of the used PVP $(3,500 \mathrm{~g} / \mathrm{mol})$. The UV/Vis extinction spectrum of the pure PVP solution is given in Figure 5c, with the measured viscosity of water and the PVP solution given in the inset. Obviously, there is no measurable extinction at the laser wavelength of $1064 \mathrm{~nm}$. 


\section{CONCLUSIONS}

Improvement of size dispersion of laser-generated colloids is often achieved in situ by anionbased charge transfer or organic ligands with high-affinity functional groups. Less is known about the quenching mechanism of macromolecular ligands, in particular non-thiolated polymers. To differentiate between the mechanisms of nanoparticle growth quenching by anions and macromolecules, we performed in situ X-ray multi-contrast imaging (with scattering contrast from a Hartmann mask) during pulsed laser ablation in liquids of gold combined with thorough ex situ evaluation. By using pure water and micromolar solutions of $\mathrm{NaCl}$ and $\mathrm{PVP}$ we have shown, that while $\mathrm{NaCl}$ reduces the size of primary and secondary nanoparticles already inside the gas phase of the laser-induced cavitation bubble, PVP does not change the nanoparticle size with respect to the sizes found in pure water. The maximum of size sensitivity in XHI is at $55 \mathrm{~nm}$, which allows for the conclusion that the organic ligand PVP does not significantly alter the size of secondary nanoparticles, composed of large solid spheres and aggregates, inside the cavitation bubble. By studying the influences of the electrolyte $\mathrm{NaCl}$ and the macromolecular ligand PVP on the size and size distributions obtained ex situ, i.e., after final collapse of the cavitation bubble, it is found that PVP does reduce the size of small primary nanoparticles $(\mathrm{d}<10 \mathrm{~nm})$ and also the degree of aggregation. In general, the concentration of PVP must exceed a threshold to effectively reduce the size of aggregates compared to pure water. The used concentration of $0.5 \mathrm{mM}$ is well above that threshold. One major difference between the additives $\mathrm{NaCl}$ and PVP is their impact on large secondary spheres. The combined results point towards the conclusion that PVP interacts with nanoparticles produced by LAL by a different mechanism as $\mathrm{NaCl}$ (as large particle size quenching is not observed). It is also indicated that 
primary and secondary particles are formed by different ablation mechanisms as large spheres can be affected by PVP ex situ more efficiently than small particles, possibly via coalescence quenching in colloidal state.

\title{
ASSOCIATED CONTENT
}

\section{Supporting Information}

The following file is available free of charge.

Influence of the effective laser fluence on the nanoparticle size distribution (Fig. S1); UV/vis calibration graph for determination of gold concentrations and representative UV/Vis extinction spectra (Fig. S2); schema of the setup for shadowgraphic imaging (Fig. S3); image sequences highlighting the maximum extension (Fig. S4) and the evolution of the cavitation bubble in pure water and in PVP solution (Fig. S5-S6); in situ SAXS-histogram of gold nanoparticles in water and salt solution (Fig. S7); surface-weighted hydrodynamic size distributions of gold in NVP, PVP, and $\mathrm{NaCl}$ (Fig. S8), TEM images of gold nanoparticle obtained in pure water, $\mathrm{NaCl}, \mathrm{PVP}$, and NVP solutions (Fig. S9-S12). (PDF)

\author{
AUTHOR INFORMATION \\ Corresponding Author \\ *e-mail: bilal.goekce@uni-due.de \\ *anton.plech@kit.edu
}

\section{Author Contributions}


Alexander Letzel, Stefan Reich, and Anton Plech conducted the in situ experiments at the ESRF synchrotron. Stefan Reich and Anton Plech performed data acquisition and interpretation. Stefan Reich performed deconvolution of the different contrast from XHI. Alexander Rack and Margie Olbinado were the local ESRF contact persons and assisted in local setup and data handling. Tomy dos Santos Rolo contributed in the planning phase and further discussions after in situ data acuisition. Alexander Kanitz and Jan Hoppius produced the Hartmann mask under the supervision of Andreas Ostendorf. The ex situ experiments and data interpretation were done by Alexander Letzel. Stephan Barcikowski, Bilal Gökce, and Anton Plech are responsible for the general conception and design of the study.

All authors have read and given approval to the final version of the manuscript.

tThe authors Alexander Letzel and Stefan Reich contributed equally.

\section{Funding Sources}

German Research Foundation DFG (BA3580/15-2 and PL325/8-2). Helmholtz Association within the program "Structure: From matter to materials and life".

\section{ACKNOWLEDGMENT}

Funding by the German Research Foundation DFG (BA3580/15-2 and PL325/8-2) and the Helmholtz Association within the program "Structure: From matter to materials and life" is gratefully acknowledged. We also thank the ESRF (Grenoble, France) and KARA (Karlsruhe, Germany) for providing the beam time, the latter for test measurements. We would further like to thank Jurij Jakobi for execution of the transmission electron microscopy analysis.

\section{ABBREVIATIONS}


AGR, additive-to-gold ratio; LAL, laser ablation in liquids; NVP: N-vinylpyrrolidone; PVP, polyvinylpyrrolidone; XHI, X-ray Hartmann mask imaging

\section{REFERENCES}

[1] A. Fojtik, A. Henglein, Laser Ablation of Films and Suspended Particles in a Solvent: Formation of Cluster and Colloid Solution, Ber. Bunsenges. Phys. Chem. 97 (1993) 252-254. [2] D. Zhang, B. Gökce, S. Barcikowski, Laser Synthesis and Processing of Colloids: Fundamentals and Applications, Chem._Rev. 117 (2017) 3990-4103.

[3] V. Merk, C. Rehbock, F. Becker, U. Hagemann, H. Nienhaus, S. Barcikowski, In Situ Non-DLVO Stabilization of Surfactant-Free, Plasmonic Gold Nanoparticles: Effect of Hofmeister's Anions, Langmuir 30 (2014) 4213-4222.

[4] C. Rehbock, V. Merk, L. Gamrad, R. Streubel, S. Barcikowski, Size control of laserfabricated surfactant-free gold nanoparticles with highly diluted electrolytes and their subsequent bioconjugation, Phys. Chem. Chem. Phys. 15 (2013) 3057-3067.

[5] G. Marzun, J. Nakamura, X. Zhang, S. Barcikowski, P. Wagener, Size control and supporting of palladium nanoparticles made by laser ablation in saline solution as a facile route to heterogeneous catalysts, Appl.Surf. Sci. 348 (2015) 75-84.

[6] M. Fischer, J. Hormes, G. Marzun, P. Wagener, U. Hagemann, S. Barcikowski, In Situ Investigations of Laser-Generated Ligand-Free Platinum Nanoparticles by X-ray Absorption Spectroscopy: How Does the Immediate Environment Influence the Particle Surface?, Langmuir 32 (2016) 8793-8802. 
[7] A. Letzel, B. Gökce, P. Wagener, S. Ibrahimkutty, A. Menzel, A. Plech, S. Barcikowski, Size Quenching during Laser Synthesis of Colloids Happens Already in the Vapor Phase of the Cavitation Bubble, J.Phys. Chem.C 121 (2017) 5356-5365.

[8] T. Tsuji, Y. Tsuboi, N. Kitamura, M. Tsuji, Microsecond-resolved imaging of laser ablation at solid-liquid interface: investigation of formation process of nano-size metal colloids, Appl.Surf. Scj. 229 (2004) 365-371.

[9] R. Tanabe, T.T.P. Nguyen, T. Sugiura, Y. Ito, Bubble dynamics in metal nanoparticle formation by laser ablation inliquid studied through high-speed laser stroboscopic videography, Anol.Surf. Scj. 351 (2015) 327-331.

[10] J. Lam, J. Lombard, C. Dujardin, G. Ledoux, S. Merabia, D. Amans, Dynamical study of bubble expansion following laser ablation in liquids, Anpl.Phys._Lett. 108 (2016) 074104.

[11] A. De Giacomo, M. Dell'Aglio, F. Colao, R. Fantoni, Double pulse laser produced plasma on metallic target in seawater: basic aspects and analytical approach, Spectrochimica Acta Part B: Atomic Snectroscopv 59 (2004) 1431-1438.

[12] A. Matsumoto, A. Tamura, T. Honda, T. Hirota, K. Kobayashi, S. Katakura, N. Nishi, K.i. Amano, K. Fukami, T. Sakka, Transfer of the Species Dissolved in a Liquid into Laser Ablation Plasma: An Approach Using Emission Spectroscopy, J. Phys. Chem. C 119 (2015) 26506-26511.

[13] S. Ibrahimkutty, P. Wagener, A. Menzel, A. Plech, S. Barcikowski, Nanoparticle formation in a cavitation bubble after pulsed laser ablation in liquid studied with high time resolution small angle x-ray scattering, Apol._Phys.Lett. 101 (2012) 103104.

[14] M.R. Gavrilović, Impact of the cavitation bubble on a plasma emission following laser ablation in liquid, Eur. Phys.J.D 71 (2017) 316. 
[15] A. Tamura, T. Sakka, K. Fukami, Y.H. Ogata, Dynamics of cavitation bubbles generated by multi-pulse laser irradiation of a solid target in water, Appl.Phys. A 112 (2013) 209-213.

[16] T. dos Santos Rolo, S. Reich, D. Karpov, S. Gasilov, D. Kunka, E. Fohtung, T.

Baumbach, A. Plech, A Shack-Hartmann Sensor for Single-Shot Multi-Contrast Imaging with Hard X-rays, Apolied Sciences 8 (2018) 737.

[17] S. Reich, J. Göttlicher, A. Letzel, B. Gökce, S. Barcikowski, T. dos Santos Rolo, T.

Baumbach, A. Plech, X-ray spectroscopic and stroboscopic analysis of pulsedlaser ablation of Zn and its oxidation, Appl. Phys. A 124 (2018) 71.

[18] S. Ibrahimkutty, P. Wagener, T. dos Santos Rolo, D. Karpov, A. Menzel, T. Baumbach, S. Barcikowski, A. Plech, A hierarchical view on material formation during pulsed-laser synthesis of nanoparticles in liquid, Sci. Rep. (2015) 5:16313.

[19] S. Reich, P. Schönfeld, A. Letzel, S. Kohsakowski, M. Olbinado, B. Gökce, S.

Barcikowski, A. Plech, Fluence Threshold Behaviour on Ablation and Bubble Formation in Pulsed Laser Ablation in Liquids, ChemPhysChem 18 (2017) 1084-1090.

[20] S. Besner, A.V. Kabashin, F.M. Winnik, M. Meunier, Synthesis of Size-Tunable PolymerProtected Gold Nanoparticles by Femtosecond Laser-Based Ablation and Seed Growth, The Journal of Physical Chemistrv C 113 (2009) 9526-9531.

[21] R. Zamiri, B.Z. Azmi, M. Darroudi, A.R. Sadrolhosseini, M.S. Husin, A.W. Zaidan, M.A. Mahdi, Preparation of starch stabilized silver nanoparticles with spatial self-phase modulation properties by laser ablation technique, Applied Phvsics A 102 (2011) 189-194. [22] F. Spano, A. Massaro, L. Blasi, M. Malerba, R. Cingolani, A. Athanassiou, In Situ Formation and Size Control of Gold Nanoparticles into Chitosan for Nanocomposite Surfaces with Tailored Wettability, Langmuir 28 (2012) 3911-3917. 
[23] S. Petersen, J. Jakobi, S. Barcikowski, In situ bioconjugation - Novel laser based approach to pure nanoparticleconjugates, Anol. Surf. Sci. 255 (2009) 5435-5438.

[24] H. Usui, Y. Shimizu, T. Sasaki, N. Koshizaki, Photoluminescence of ZnO Nanoparticles Prepared by Laser Ablation in Different Surfactant Solutions, The Journal of Physical Chemistry B 109 (2005) 120-124.

[25] S. Salmaso, P. Caliceti, V. Amendola, M. Meneghetti, J.P. Magnusson, G. Pasparakis, C. Alexander, Cell up-take control of gold nanoparticles functionalized with a thermoresponsive polymer, J.Mater. Chem. 19 (2009) 1608-1615.

[26] J. Tomko, J.J. Naddeo, R. Jimenez, Y. Tan, M. Steiner, J.M. Fitz-Gerald, D.M. Bubb, S.M. O'Malley, Size and polydispersity trends found in gold nanoparticles synthesized by laser ablation in liquids, Phys. Chem. Chem. Phys. 17 (2015) 16327-16333.

[27] D. Amans, C. Malaterre, M. Diouf, C. Mancini, F. Chaput, G. Ledoux, G. Breton, Y. Guillin, C. Dujardin, K. Masenelli-Varlot, P. Perriat, Synthesis of Oxide Nanoparticles by Pulsed Laser Ablation in Liquids Containing a Complexing Molecule: Impact on Size Distributions and Prepared Phases, L. Phys.Chem.C 115 (2011) 5131-5139.

[28] R. Singh, R.K. Soni, Laser synthesis of aluminium nanoparticles in biocompatible polymer solutions, Applied Phvsics A 116 (2014) 689-701.

[29] T. Tsuji, D.-H. Thang, Y. Okazaki, M. Nakanishi, Y. Tsuboi, M. Tsuji, Preparation of silver nanoparticles by laser ablation in polyvinylpyrrolidone solutions, Appl. Surf. Sci. 254 (2008) 5224-5230.

[30] X. Liu, F. Zhnag, R. Huang, C. Pan, J. Zhu, Capping Modes in PVP-Directed Silver Nanocrystal Growth: Multi-Twinned Nanorods versus Single-Crystalline Nano-Hexapods, Cryst. Growth Des. 8 (2008) 1916-1923. 
[31] A. Mezni, T. Dammak, A. Fkiri, A. Mlayah, Y. Abid, L.S. Smiri, Photochemistry at the Surface of Gold Nanoprisms from Surface-Enhanced Raman Scattering Blinking, J. Phys. Chem. C 118 (2014) 17956-17967.

[32] L. Xu, G. Wang, X. Zheng, H. Pan, J. Zhu, Z. Li, S.-H. Yu, Competitive Adsorption between a Polymer and Its Monomeric Analog Enables Precise Modulation of Nanowire Synthesis, Chem 4 (2018) 2451-2462.

[33] R. Seoudi, A.A. Fouda, Elmenshawy, Synthesis, characterization and vibrational spectroscopic studies of different particle size of gold nanoparticle capped with polyvinylpyrrolidone, Phvsica B 405 (2010) 906-911.

[34] H.L. Liu, P. Hou, Low-temperature synthesis and characterization of PVP-capped FeAu nanoparticles, J.Mater. Res. 26 (2011) 2040-2049.

[35] P. Abdulkin, T.L. Precht, B.R. Knappett, H.E. Skelton, D.A. Jefferson, A.E.H. Wheatley, Systematic Control of Size and Morphology in the Synthesis of Gold Nanoparticles, Part. Part. Syst. Chem. 31 (2014) 571-579.

[36] D.P. Stankus, S.E. Lohse, J.E. Hutchison, J.A. Nason, Interactions between Natural Organic Matter and Gold Nanoparticles Stabilized with Different Organic Capping Agents, Environ. Sci. Technol. 45 (2011) 3238-3244.

[37] K.I. Requejo, A.V. Liopo, P.J. Derry, E.R. Zubarev, Accelerating Gold Nanorod Synthesis with Nanomolar Concentrations of Poly(vinylpyrrolidone), Langmuir 33 (2017) 12681-12688.

[38] T. Tsuji, M. Nakanishi, T. Mizuki, S. Ozono, M. Tsuji, Y. Tsuboi, Preparation and Shape-Modification of Silver Colloids by Laser Ablation in Liquids: A Brief Review, Sci. Adv. Mater. 4 (2012) 391-400. 
[39] C.L. Sajti, S. Petersen, A. Menéndez-Manjón, S. Barcikowski, In-situ bioconjugation in stationary media and in liquid flow by femtosecond laser ablation, Appl. Phys. A 101 (2010) $259-264$.

[40] C.L. Sajti, A. Barchanski, P. Wagener, S. Klein, S. Barcikowski, J. Phys. Chem. C 115 (2011) 5094-5101.

[41] S. Jendrzej, B. Gökce, V. Amendola, S. Barcikowski, Barrierless growth of precursorfree, ultrafast laser-fragmented noble metal nanoparticles by colloidal atom clusters - A kinetic in situ study, I. Colloid. Interface Sci. 463 (2016) 299-307.

[42] P. Wagener, S. Ibrahimkutty, A. Menzel, A. Plech, S. Barcikowski, Dynamics of silver nanoparticle formation and agglomeration inside the cavitation bubble after pulsed laser ablation in liquid, Phys. Chem. Chem. Phys. 15 (2013) 3068-3074.

[43] H.H. Wen, E.E. Bennett, M.M. Hedegus, S.C. Carroll, Spatial Harmonic Imaging of Xray Scattering_Initial Results, IEEE T. Med. Imaging 27 (2008) 997-1002.

[44] Y. Liu, B. Ahr, A. Linkin, G.J. Diebold, C. Rose-Petruck, X-ray spatial harmonic imaging of phase objects, Opt._Lett. 36 (2011) 2209-2211.

[45] S.K. Lynch, V. Pai, J. Auxier, A.F. Stein, E.E. Bennett, C.K. Kamble, X. Xiao, W.-K. Lee, N.Y. Morgan, H.H. Wen, Interpretation of dark-field contrast and particle-size selectivity in grating interferometers, Appl. Opt. 50 (2011) 4310-4319.

[46] A.F. Stein, J. Ilavsky, R. Kopace, E.E. Bennett, H. Wen, Selective imaging of nanoparticle contrast agents by a single-shot $x$-ray diffraction technique, Opt. Express 18 (2010) $13271-13278$. 
[47] F.A. Vittoria, M. Endrizzi, P.C. Diemoz, A. Zamir, U.H. Wagner, C. Rau, I.K. Robinson, A. Olivio, X-ray absorption, phase and dark-field tomography through a beam tracking approach, Sci.Rep. 5 (2015) 16318.

[48] G.M. Hale, M.R. Querry, Optical Constants of Water in the 200-nm to 200- $\mu \mathrm{m}$ Wavelength Region, Applied Optics 12 (1973) 555-563.

[49] T. Wagner, thorstenwagner/ij-particlesizer: ParticleSizer 1.0.7, in, https://zenodo.org/record/163568\#.WoqZWXwiFpg, 2016.

[50] H.H. Wen, E.E. Bennett, R. Kopace, A.F. Stein, V. Pai, Single-shot x-ray differential phase-contrast and diffraction imaging using two-dimensional transmission gratings, Opt. Lett. 35 (2010) 1932-1934.

[51] S. Reich, T. dos Santos Rolo, A. Letzel, T. Baumbach, A. Plech, Scalable, large area compound array refractive lens for hard X-rays, Applied Physics Letters 112 (2018) 151903. [52] S. Kaeppler, F. Bayer, T. Weber, A. Maier, G. Anton, J. Hornegger, M. Beckmann, P.A. Fasching, A. Hartmann, F. Heindl, T. Michel, G. Oezguel, G. Pelzer, C. Rauh, J. Rieger, R. Schulz-Wendtland, M. Uder, D. Wachter, E. Wenkel, C. Riess, Signal decomposition for X-ray dark-field imaging, Med. Image. Comput. Comput. Assist. Interv. 17 (2014) 170-177. [53] G.-R. Zhang, B.-Q. Xu, Surprisingly strong effect of stabilizer on the properties of Au nanoparticles and $P t^{\wedge} A u$ nanostructures in electrocatalysis, Nanoscale 2 (2010) 2798-2804. [54] H. Tsunoyama, N. Ichikuni, H. Sakurai, T. Tsukuda, Effect of Electronic Structures of Au Clusters Stabilized by Poly(N-vinyl-2-pyrrolidone) on Aerobic Oxidation Catalysis, Journal of the American Chemical Society 131 (2009) 7086-7093. 
[55] M. Okumura, Y. Kitagawa, T. Kawakami, M. Haruta, Theoretical investigation of the hetero-junction effect in PVP-stabilized Au13 clusters. The role of PVP in their catalytic activities, Chemical Physics Letters 459 (2008) 133-136.

[56] H. Wang, X. Qiao, J. Chen, X. Wang, S. Ding, Mechanisms of PVP in the preparation of silver nanoparticles, Mater. Chem. Phys. 94 (2005) 449-453.

[57] F. Pfeiffer, M. Bech, O. Bunk, P. Kraft, E.F. Eikenberry, C. Brönnimann, C. Grünzweig, C. David, Hard-X-ray dark-field imaging using a grating interferometer, Nat. Mater. 7 (2008) 134-137.

[58] A. Letzel, B. Gökce, A. Menzel, A. Plech, S. Barcikowski, Primary particle diameter differentiation and bimodality identification by five analytical methods using gold nanoparticle size distributions synthesized by pulsed laser ablation in liquids, Appl. Surf. Sci. 435 (2018) 743751.

[59] P. Wagener, G. Brandes, A. Schwenke, S. Barcikowski, Impact of in situ polymer coating on particle dispersion into solid laser-generated nanocomposites, Physical Chemistry Chemical Physics 13 (2011) 5120-5126. 


\section{TABLE OF CONTENTS}

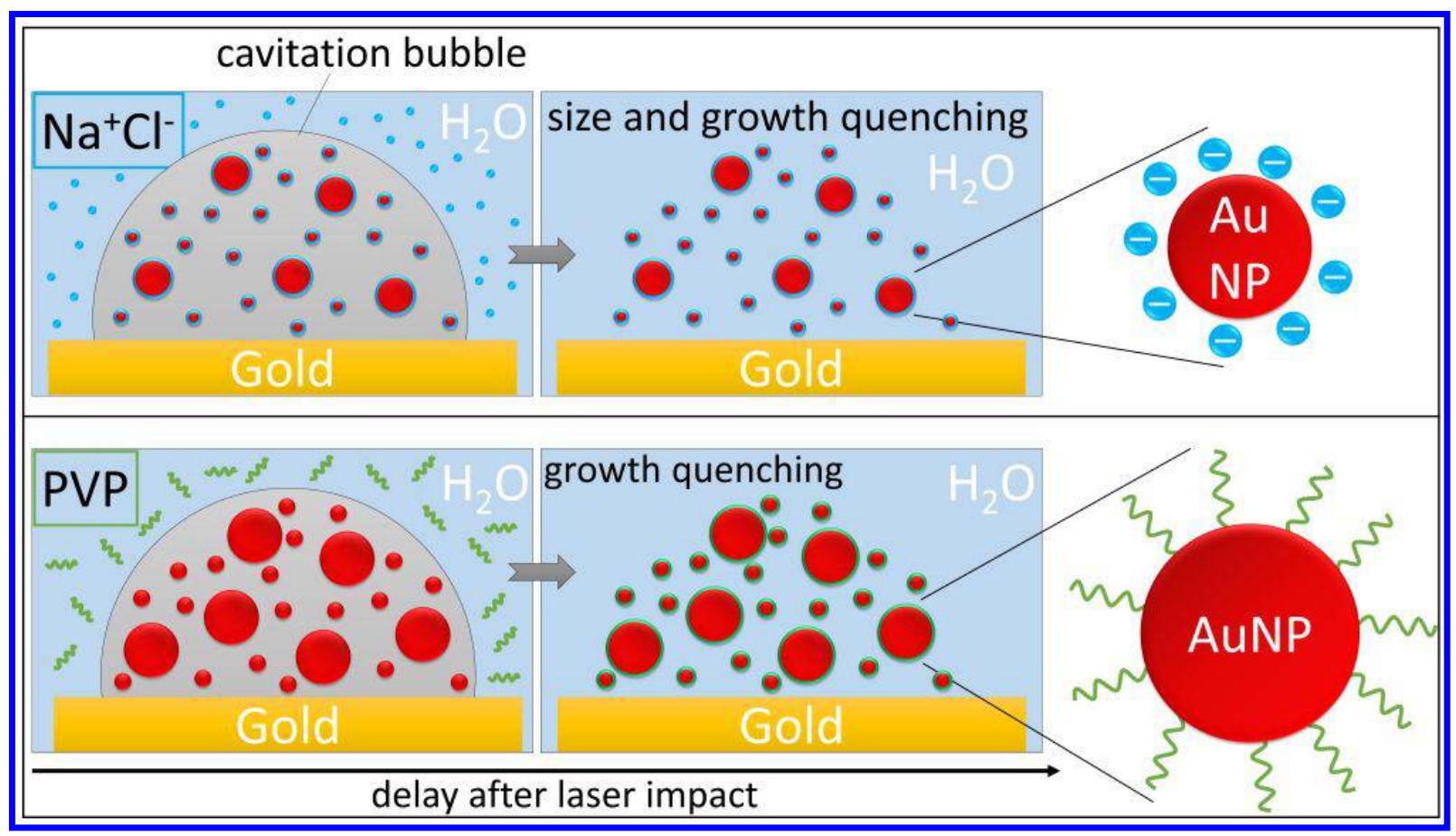




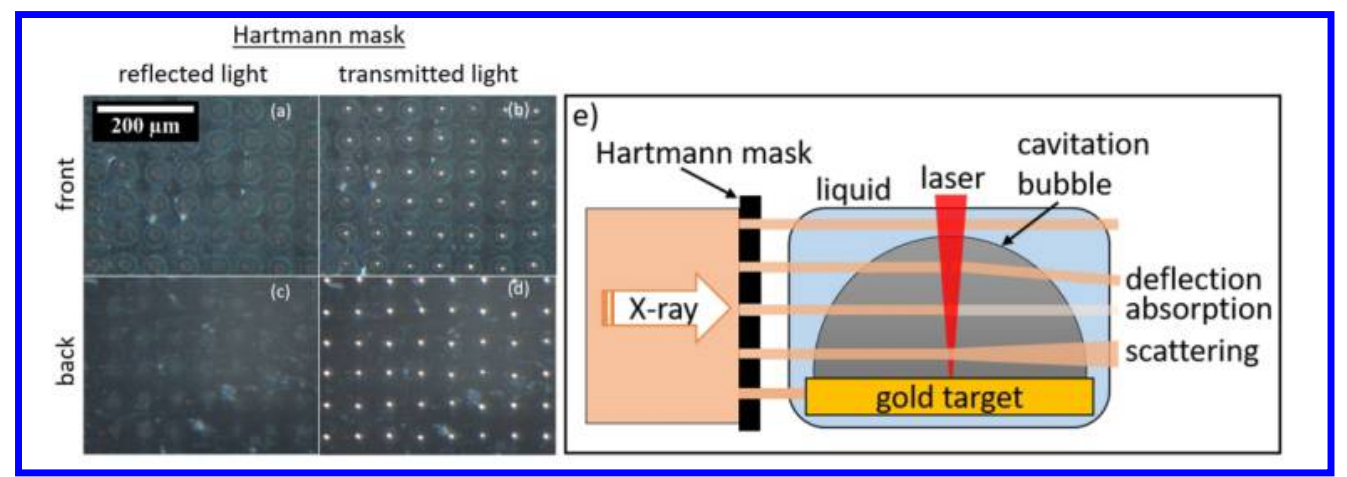

Figure 1. Images of the Pt foil acting as a Hartmann mask for in situ experiments obtained by optical microscopy. Front of the mask in reflected (a) and transmitted light mode (b). The laser-drilled holes are funnel-shaped on the front side (side at which the laser beam impinges). Back of the mask in reflected (c) and transmitted light mode (d). Sketch of the XHI approach with the incoming X-ray beam being split into multiple beamlets by the Hartmann mask, and undergoing absorption, differential phase shift (deflection) and diffuse scattering (broadening) when crossing the cavitation bubble and the confined nanoparticles (e).

$160 \times 54 \mathrm{~mm}(300 \times 300 \mathrm{DPI})$ 

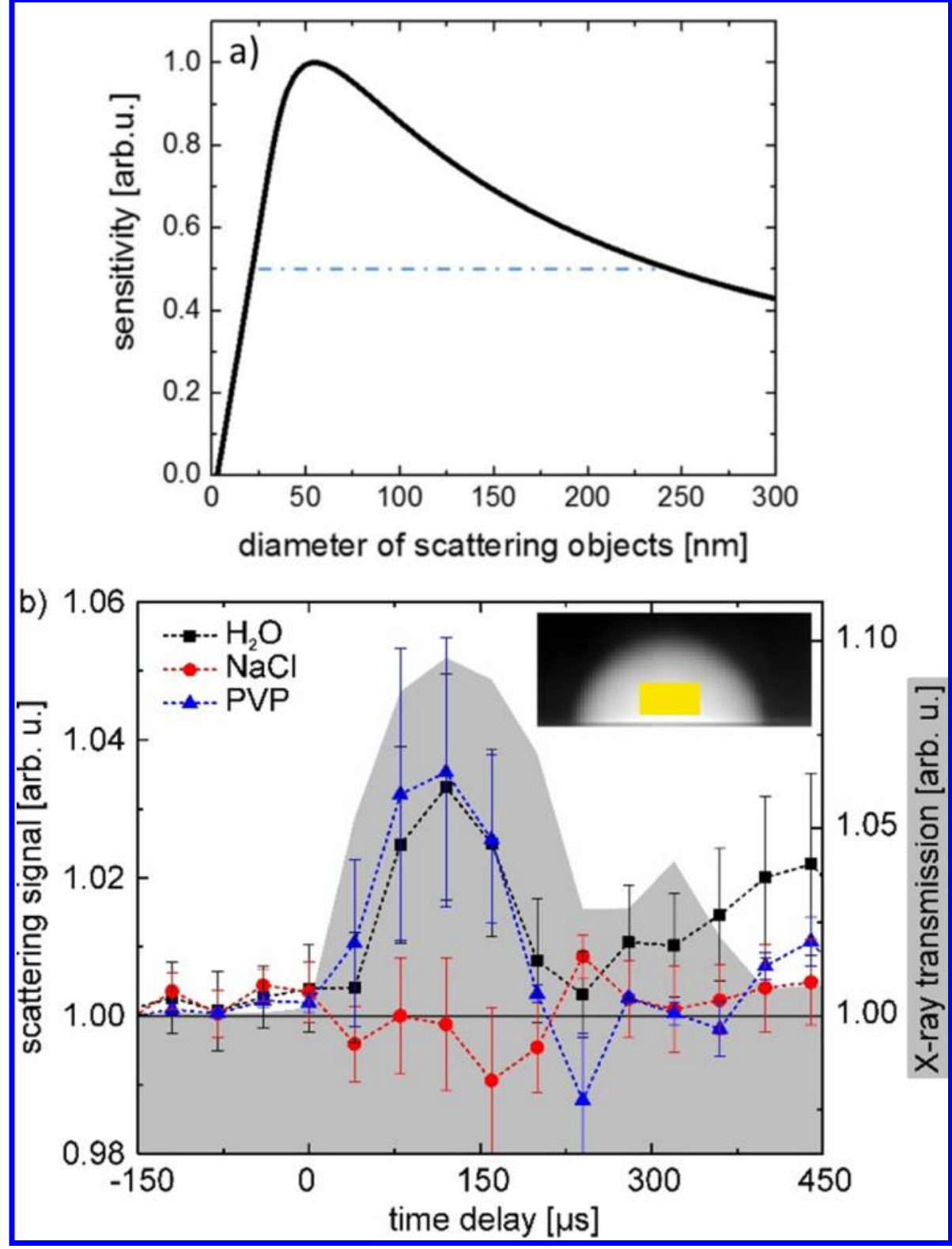

Figure 2. Scattering sensitivity on nanoparticles as function of the scattering object size for the given setup. The blue dashed-dotted line indicates the size interval above $50 \%$ relative sensitivity (a). Scattering signal as function of the delay after laser impact obtained at the center of the cavitation bubble (inset shows the transmission signal together with the region of interest) (b). Scattering signals of gold nanoparticles in pure water (black squares), $\mathrm{NaCl}$ - (red circles) and PVP-solution (blue triangle) (both $0.5 \mathrm{mM}$ ). The shaded curve represents the change in $\mathrm{X}$-ray transmission starting from 1 ( $0 \%$ intensity change) before laser arrival. Error bars are the standard deviation between several runs.

\section{$80 \times 106 \mathrm{~mm}(300 \times 300$ DPI $)$}


c)
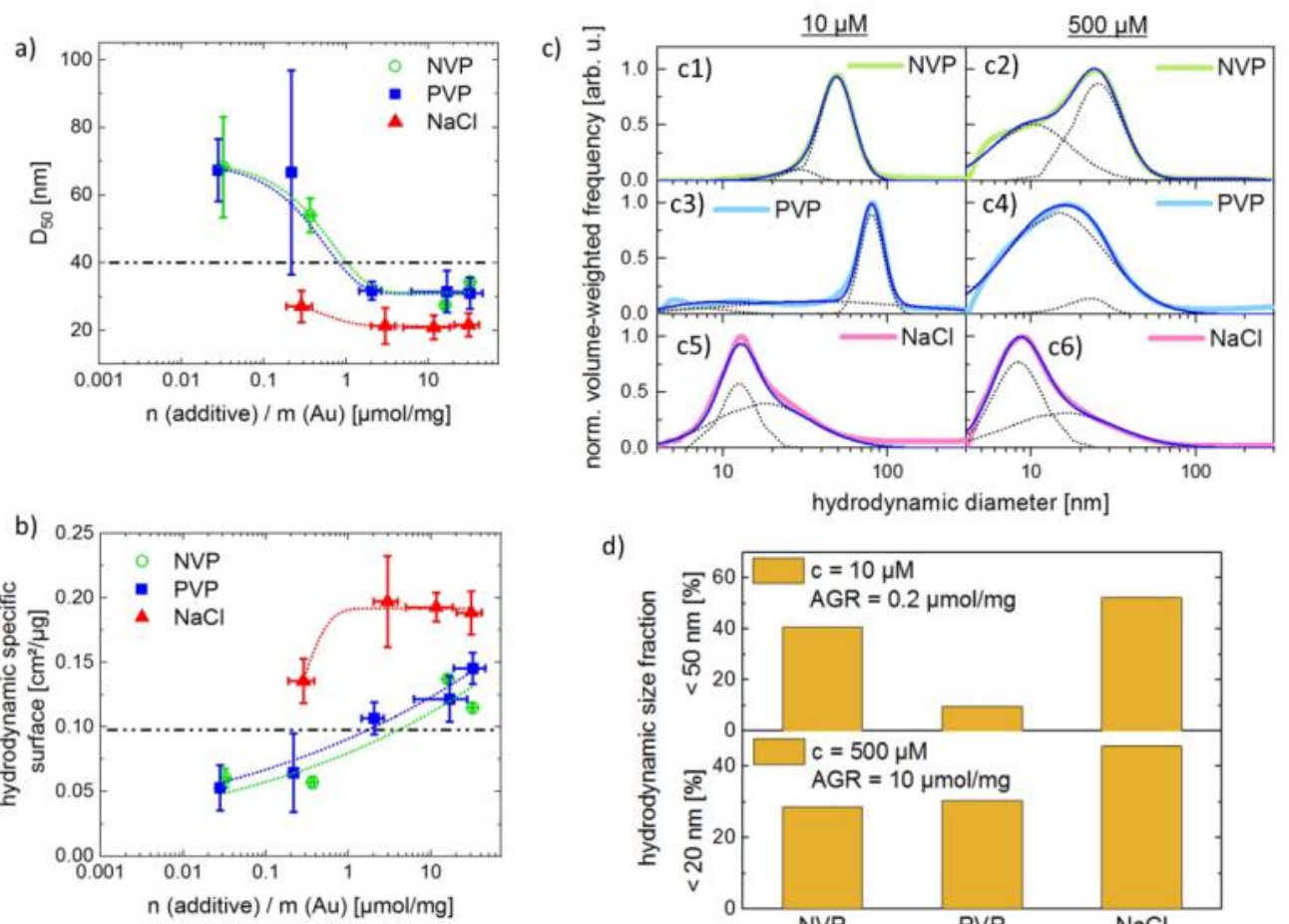

d)

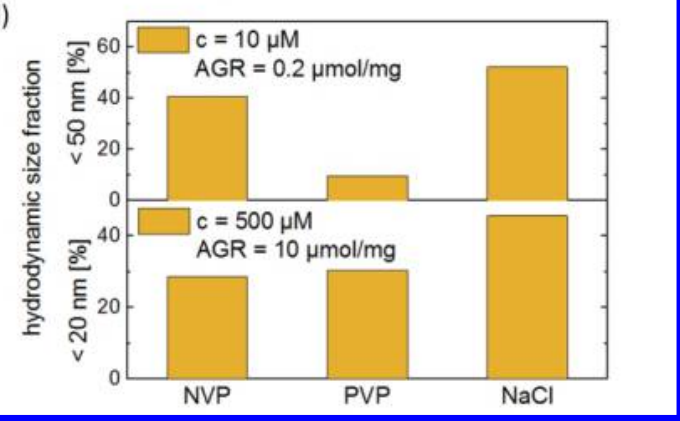

Figure 3. (a) Volume-weighted hydrodynamic median diameter obtained by analytical disc centrifugation and

(b) related specific surface area of gold nanoparticles in PVP and $\mathrm{NaCl}$ as function of the additive-to-gold ratio (AGR). The dashed-dotted horizontal lines mark the reference experiments from LAL in pure water. Nanoparticle mass concentrations are obtained by optical UV/Vis extinction spectroscopy. (c) Volumeweighted particle size distributions of colloids in low and high additive concentrations derived from ADC analysis. (d) Hydrodynamic size fractions of particles $<20 \mathrm{~nm}$ and $<50 \mathrm{~nm}$. The data is derived from the ADC size distributions in (c). The results display the properties of the particles in their nascent hydrodynamic environment.

$160 \times 115 \mathrm{~mm}(300 \times 300 \mathrm{DPI})$ 
Figure 4. Representative TEM histograms of AuNP in pure water, $\mathrm{NaCl}$ and PVP at AGRs of 0.2 and 10 $\mu \mathrm{mol} / \mathrm{mg}$ (left column). The envelopes shown as solid red lines are obtained by lognormal fitting and the center of gravity $X_{c}$ is displayed. The middle column shows the calculated volume-weighted histograms of the same samples with corresponding median diameters D50. In the right column the volume-weighted histograms are further weighted by the XHI sensitivity curve shown in Figure $2 a$. The plot in the lower right corner sums up the volume-weighted primary particle median diameters as a function of the type and AGR of additive.

$160 \times 111 \mathrm{~mm}(300 \times 300 \mathrm{DPI})$ 

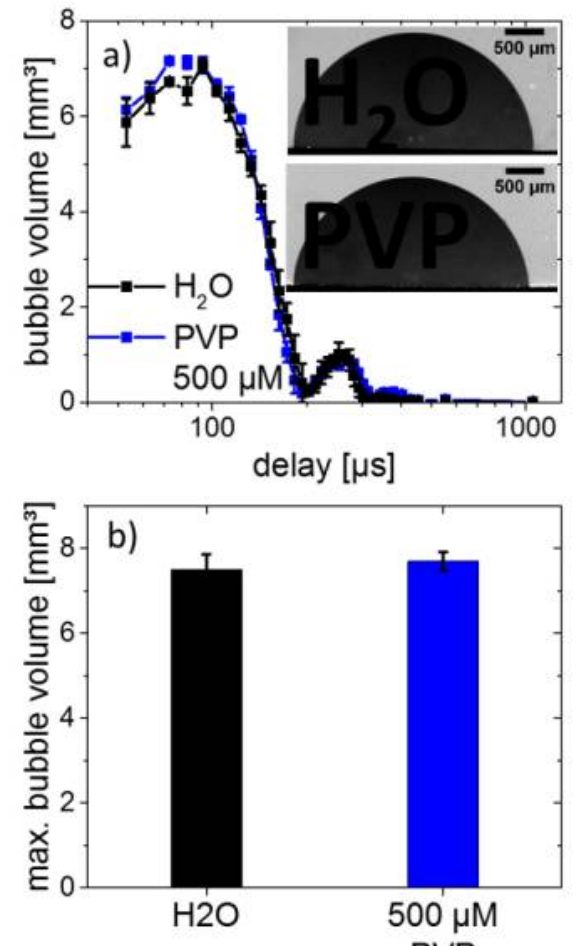

PVP

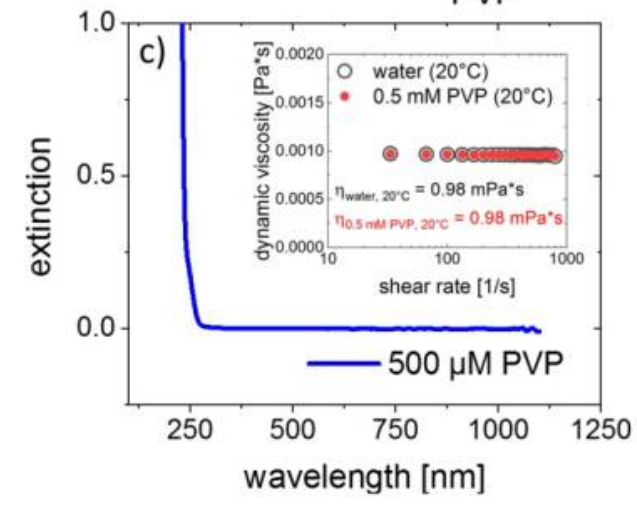

Figure 5. (a) Cavitation bubble dynamics as function of the delay from the laser impact. The PVP molecules do not affect the dynamics of the bubble.(b) Results of image analysis of the cavitation bubble at its maximum extension at $93 \mu \mathrm{s}$ after laser impact on the target showing no difference between pure water and $500 \mu \mathrm{M}$ PVP solution. (c) UV/Vis extinction spectrum of a $500 \mu \mathrm{M}$ PVP solution. The solution is transparent at the laser wavelength of $1064 \mathrm{~nm}$. Inset: Dynamic viscosity of pure water and a $0.5 \mathrm{mM}$ PVP solution.

$$
80 \times 170 \mathrm{~mm}(300 \times 300 \mathrm{DPI})
$$

